\title{
Patient Preference and Satisfaction with the Use of Telemedicine for Glycemic Control in Patients with Type 2 Diabetes: A Review
}

This article was published in the following Dove Press journal: Patient Preference and Adherence

\author{
Ruth Sim (D) \\ Shaun Wen Huey Lee (iD) 2,3 \\ 'School of Pharmacy, Monash University \\ Malaysia, Subang Jaya, Selangor, Malaysia; \\ ${ }^{2}$ School of Pharmacy, Faculty of Health \\ and Medical Sciences, Taylor's University, \\ Subang Jaya, Selangor, Malaysia; ${ }^{3}$ Asian \\ Centre for Evidence Synthesis in \\ Population, Implementation and Clinical \\ Outcomes (PICO), Health and Well- \\ Being Cluster, Global Asia in the 21 st \\ Century (GA2I) Platform, Monash \\ University Malaysia, Bandar Sunway, \\ Selangor, Malaysia
}

Background: Telemedicine has the potential to improve patient care and management for various chronic diseases such as type 2 diabetes. To ensure the success of any telemedicine program, there is a need to understand the patients' satisfaction and their preferences. This review aims to collate and provide evidence related to practices that may influence the performance of telemedicine for patients with type 2 diabetes.

Methods: We searched three electronic databases for studies examining patients' satisfaction and preferences for using telemedicine in type 2 diabetes. An evaluation matrix was developed to collect the data from the included articles. A total of 20 articles were identified and data on the key outcomes identified were narratively synthesized.

Results: Patients were generally satisfied with the use of telemedicine for management of type 2 diabetes. Users reported that telemedicine was beneficial as it provided constant monitoring, improved access to healthcare providers, and reduced waiting time. When adopting a telemedicine platform, most patients expressed preference for mobile health (mHealth) as the telemedicine modality, especially if it has been endorsed by their physician. To improve usability and sustainability, patients suggested that modules related to diabetes education be enhanced, together with sufficient technical and physician support when adopting telemedicine. Patients also expressed the importance of having a sufficiently flexible platform that could be adapted to their needs.

Conclusion: Personalized telemedicine strategies coupled with appropriate physician endorsement greatly influences a patient's decision to undertake telemedicine. Future work should focus on improving telemedicine infrastructure and increasing physician's involvement, especially during the implementation phase.

Keywords: type 2 diabetes, satisfaction, preference, telemedicine, mHealth, review
Correspondence: Shaun Wen Huey Lee School of Pharmacy, Monash University Malaysia, Subang Jaya, Selangor, Malaysia Email shaun.lee@monash.edu

\section{Introduction}

Over the past decade, the prevalence of diabetes has increased dramatically. It is estimated that diabetes affects more than 463 million adults worldwide in 2019 , with type 2 diabetes accounting for more than $90 \%$ of the cases. ${ }^{1}$ Diabetes is now the tenth leading cause of mortality worldwide. ${ }^{2}$ This increasing prevalence of type 2 diabetes is associated with rising number of comorbidities, healthcare needs, and costs. Evidence from multiple randomized controlled studies has shown that managing glucose and diabetes related risk factors including cardiovascular disease can prevent or delay mortality by up to $33 \% .^{3-5}$ One of the biggest challenges in managing this condition is the requirement for continuous patient engagement, 
especially due to the need for regular follow-up, adherence to therapies and lifestyle modifications, as well as the disease itself, which requires a high-touch management approach. ${ }^{6}$ Self-care has been suggested to be a vital component in the prevention and management of diabetes, as this can lead to better blood glucose control. Despite these benefits, most people with diabetes find it difficult to implement the necessary changes involved in self-care, due to factors such as poor self-efficacy, geographical barriers, as well as the lack of social support.

The implementation of digital technology or, more specifically, telemedicine has grown exponentially and is now frequently used in health education promotion, disease management, and surveillance of many chronic diseases. While various definitions for telemedicine exist, it can be broadly defined as the use of technology to effectively connect individuals and their healthcare providers to support in their care. ${ }^{7}$ This can range from a simple telephone call between a doctor and the patient to complex systems involving remote monitoring with feedback and video consultations. These could be delivered through a multitude of platforms including telehealth, mHealth, remote home monitoring, social platforms, and patient portals (See Text Box for full definition). Reviews performed to date have suggested that telemedicine could potentially ease the healthcare burden borne by healthcare providers, facilitate patient self-management, and address patient needs. ${ }^{8-11}$ Due to this migration to telemedicine, patients and physicians alike are now armed with a plethora of different telemedicine modalities and applications to assist with the management of blood glucose in the treatment of type 2 diabetes.

In order to maximize patient well-being, it is of paramount importance to acknowledge and understand potential user behavior and feelings toward technology and how it will fit into their lifestyles. ${ }^{12}$ While these emerging technologies are perceived to reduce healthcare burden, the input for technology often comes from the perspective of clinicians. Past studies have focused on the healthcare provider's perspective and their preference of using telemedicine. Only limited studies have sought to understand patient satisfaction and their views regarding the use of these technologies in glucose management. This is important, given that the patient's satisfaction with telemedicine will ultimately influence their compliance with treatment regimens and their outcomes. ${ }^{13}$ Understanding patient perception helps the telemedicine provider to customize product features, and incorporate them into payer reimbursement for these strategies. This review article will attempt to summarize and provide an overview of the various telemedicine strategies used for management of type 2 diabetes. We describe the currently available technology and patient satisfaction and preference toward them.

\section{Text Box: Widely Adopted Platforms to Deliver Telemedicine ${ }^{10,14}$ \\ Telehealth}

Provision of healthcare services through use of information and communication technologies (ICT) rather than traditional face-to-face communication between patients and physicians. Modes of delivery include telephone calls and video conferencing.

\section{mHealth}

Use of mobile and wireless technologies to achieve health goals. These can include traditional core functions of textmessaging as well as more complex forms of digital health applications that allows monitoring of nutrition status, physical activity, and glucose level. Some also provide tips on insulin titration and education. ${ }^{15}$

\section{Remote Home Monitoring}

Processes that monitor and collect patient health status and data from a distance, in contexts outside of conventional clinical settings. Data is then transmitted back to healthcare providers.

\section{Social Platforms}

Social media are web-based interactive technologies that facilitate information sharing via communities formed. These include commercial platforms such as Facebook, Instagram, Twitter, and specific websites for people with diabetes.

\section{Patient Portals}

Online platforms that allow patients to access their electronic health records and communicate with their health care providers. Features include viewing and uploading of clinic data, setting treatment goals, appointment scheduling, and medication refill requests. Portals enable secure communication with healthare providers and patient education. mHealth could be incorporated into a welldesigned patient portal. 


\section{Methods}

We searched MEDLINE (via PubMed), EMBASE, and Cochrane Central Register of Controlled Trials for articles from database inception up to August 12, 2020, using the search terms: "telehealth", "teleconferenc*", "telemonitor*", "videoconferenc*", "video monitoring", "internet monitoring", "video consultation", "telephone", "smartphone", "app", "sms", "text messag*", "mobile health", "mHealth", "eHealth", "portal", "game", "type 2 diabetes", "NIDDM", "T2DM", "non insulin* depend*", "satisfaction", "preference", "perception", and "experience". We also searched the reference lists of original research articles, clinical guidelines, systematic reviews, and meta-analyses for further relevant articles. Primary articles which examined the use of telemedicine and patient satisfaction in people with type 2 diabetes were eligible for inclusion. Duplicates, articles examining population solely for type 1 diabetes, gestational diabetes, or mHealth usability and perception of health professionals were excluded. The standard systematic review procedures were followed to ensure our search was comprehensive and controlled for selection bias. Among the articles screened, we included 20 articles that examined satisfaction and preference toward telemedicine among patients with type 2 diabetes (Figure 1, PRISMA flowchart). A summary of these studies can be found in Table 1 .

One author (RS) performed the data extraction of the studies using Microsoft Excel, while the second author (SLWH) validated the data. Each author then compiled notes on study findings and any important findings identified. A meeting was held between authors where observations were discussed and combined into larger recurring themes related to the key determinants or impediments to satisfaction and preference. These were sorted into an affinity matrix for satisfaction and preference. The affinity matrix contained the themes, reference number of the studies, and their frequencies (Table 2).

\section{Results \\ Overall Satisfaction}

Most patients were satisfied with the use of telemedicine for management of their condition, in both urban and rural settings alike (Figure 2). ${ }^{12,-16-27}$ Studies have identified several factors which led to higher patient satisfaction, and were related to the following themes: time-saving, access, and support. While the adoption of telemedicine was reportedly to be low among older adults due to their unfamiliarity with technology, we have seen the increase in usage and uptake of health technologies in the past decade. $^{28}$ This trend is further accelerated in the current COVID-19 pandemic, as this group, which has been conventionally labelled as "digital illiterate", is pushed to adopt this new modality, due to limited in-person care options. However, while the older adults have minimal concerns with telemedicine, they view telemedicine as an alternative to supplement clinic consultations rather than a complete substitute. ${ }^{12}$ In these studies, we did not find any concerns of trustworthiness by patients. ${ }^{12}$ While patients find it easy to follow the healthy lifestyle advices using telemedicine, they related how cumbersome it was to enter data related to their glucose levels, physical activities, and diet daily. ${ }^{29}$

\section{Round the Clock Access and Monitoring}

Telemedicine was highly valued among patients for their omnipresence, as they felt that their health conditions were constantly monitored and cared for throughout the day. ${ }^{12}$ Patients particularly liked the idea that they could receive advice from their physicians without the need to visit a healthcare facility. ${ }^{12}$ This is important for people with type 2 diabetes as there is a need to monitor blood glucose levels constantly to ensure that they are within control. Using telemedicine, patients felt secure as their health status was constantly being monitored, and they could receive feedback as needed. ${ }^{30}$ This, however, needs to take patient's personal preference into consideration, especially if they have concerns over their privacy and confidentiality. ${ }^{23}$

As such, to ensure patient compliance, there is a need to educate patients on the importance and benefits of telemedicine. The benefits include the ability to exchange data using telemedicine with healthcare personnel outside of consultation hours, ${ }^{25,29}$ trend analysis, data visualization to facilitate discussion with their doctor, ${ }^{12,22,29,31,32}$ and data storage ability. ${ }^{23}$ Indeed, patients found that these features helped them understand how their lifestyle actions affected their blood glucose in more detail. ${ }^{29}$

\section{Access to Different Healthcare Providers}

Another potential of telemedicine is the ability to reduce geographical barriers, allowing for better healthcare resource utilization, and improve access to physicians as well as resource sharing. ${ }^{12,24,25,27}$ Many individuals with type 2 diabetes often seek different healthcare providers in their course of treatments, including primary care 


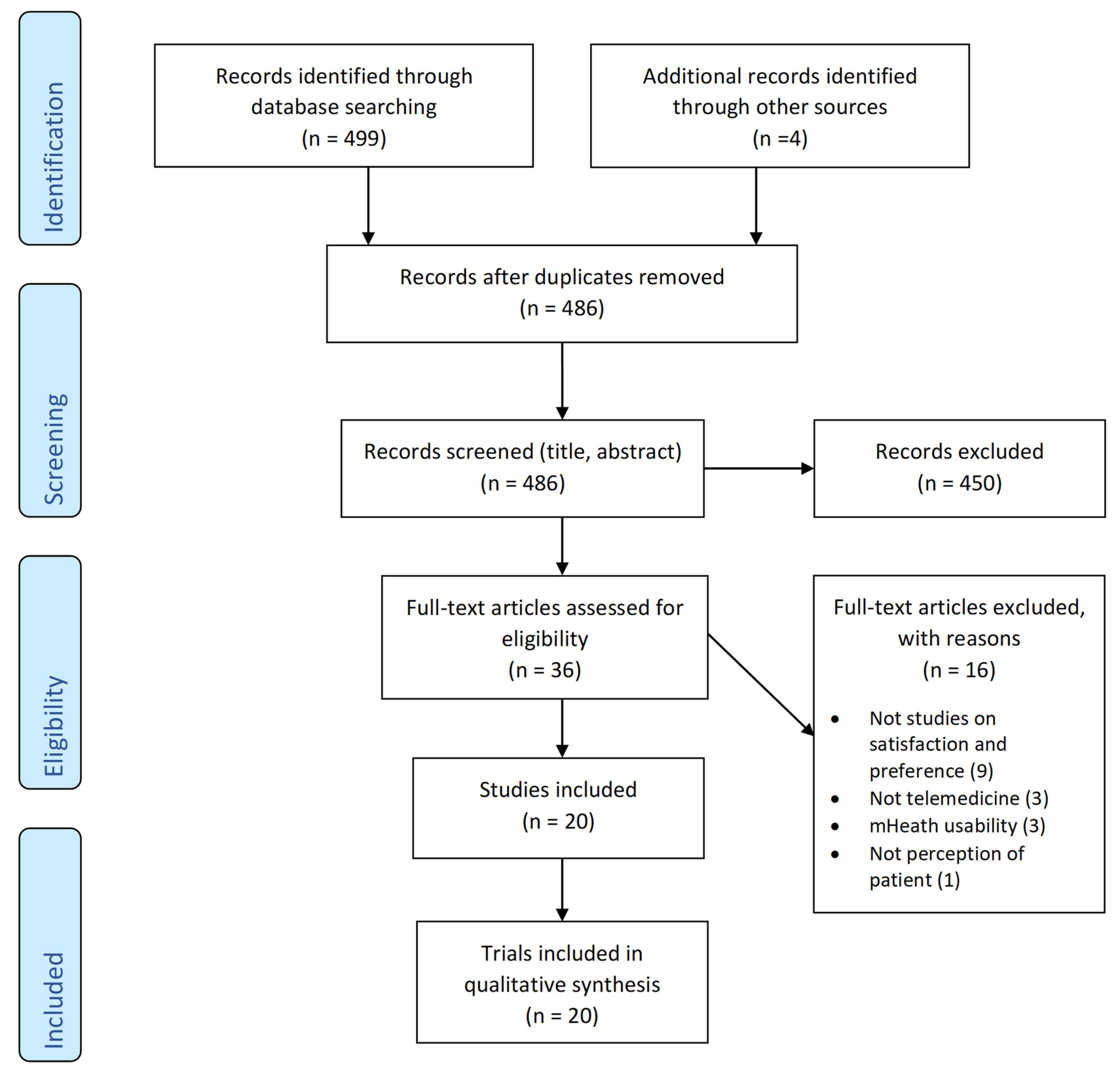

Figure I PRISMA flow diagram for literature search process.

physicians, endocrinologist, diabetologists, and cardiologists. As such, time saved from traveling to visit multiple healthcare providers can be better utilized for self-management of blood glucose. ${ }^{33}$ Patients particularly valued the ease of access to their physicians by using telemedicine, especially those with mobility issues, ${ }^{12}$ and those living in rural areas, ${ }^{22,27}$ as they could receive care which was otherwise unavailable in their locality. ${ }^{29}$ Nevertheless, one issue highlighted by patients is the lack of continuity of care provided through telemedicine. ${ }^{27}$ They reportedly noted that the quality of care delivered varied, and that there was poor communication between team members. ${ }^{12}$ In addition, patients also disliked the lag-time it took between posting their queries and responses they received. $^{12,25}$

\section{Time-Saving}

The potential time-saving with the use of telemedicine is one of the most common reasons cited by patients to embrace telemedicine. ${ }^{12,17,24,25,27}$ Indeed, due to the ease of use with such technology, patients, especially those who had to rely on their carers to attend any healthcare 


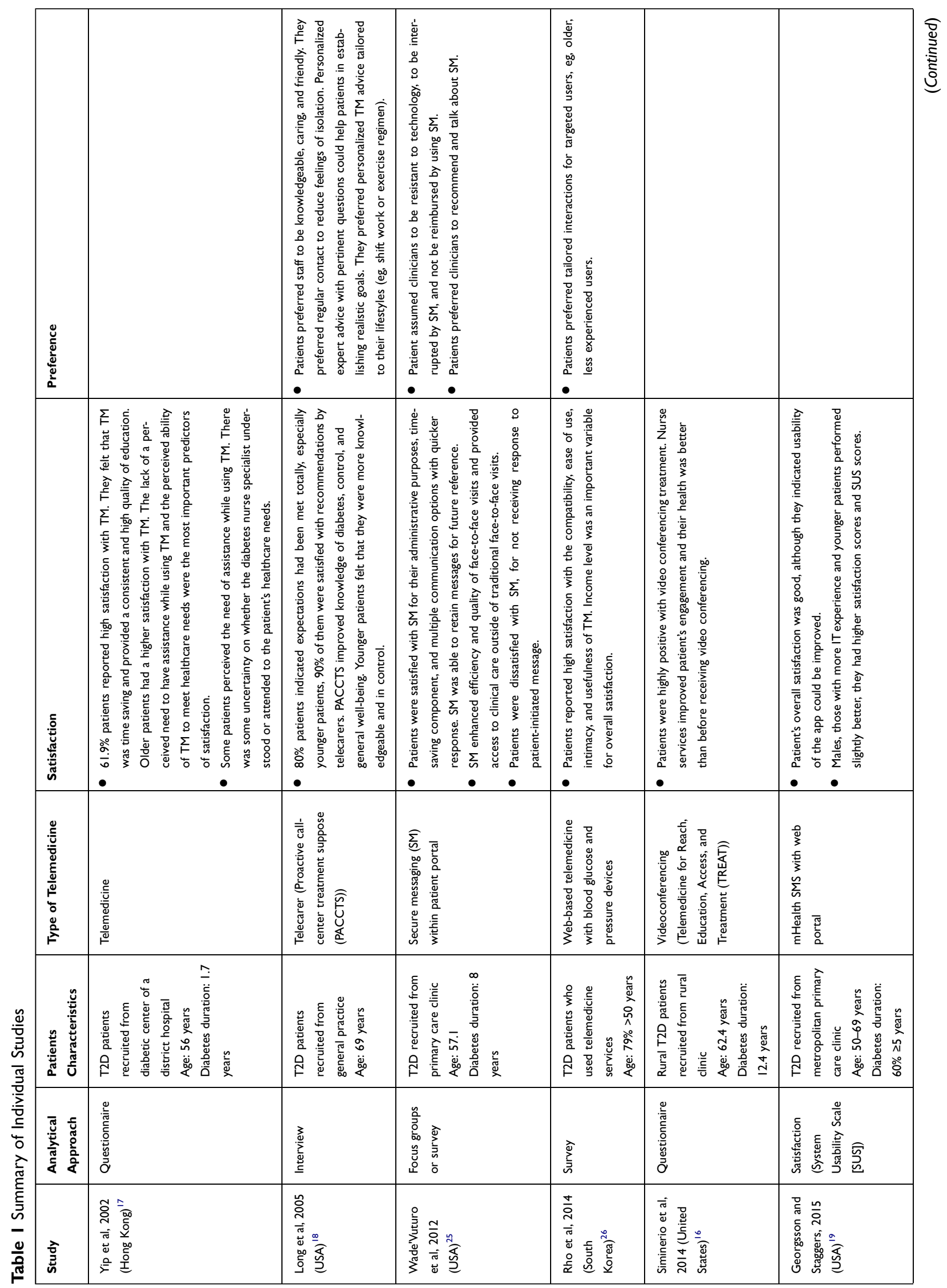




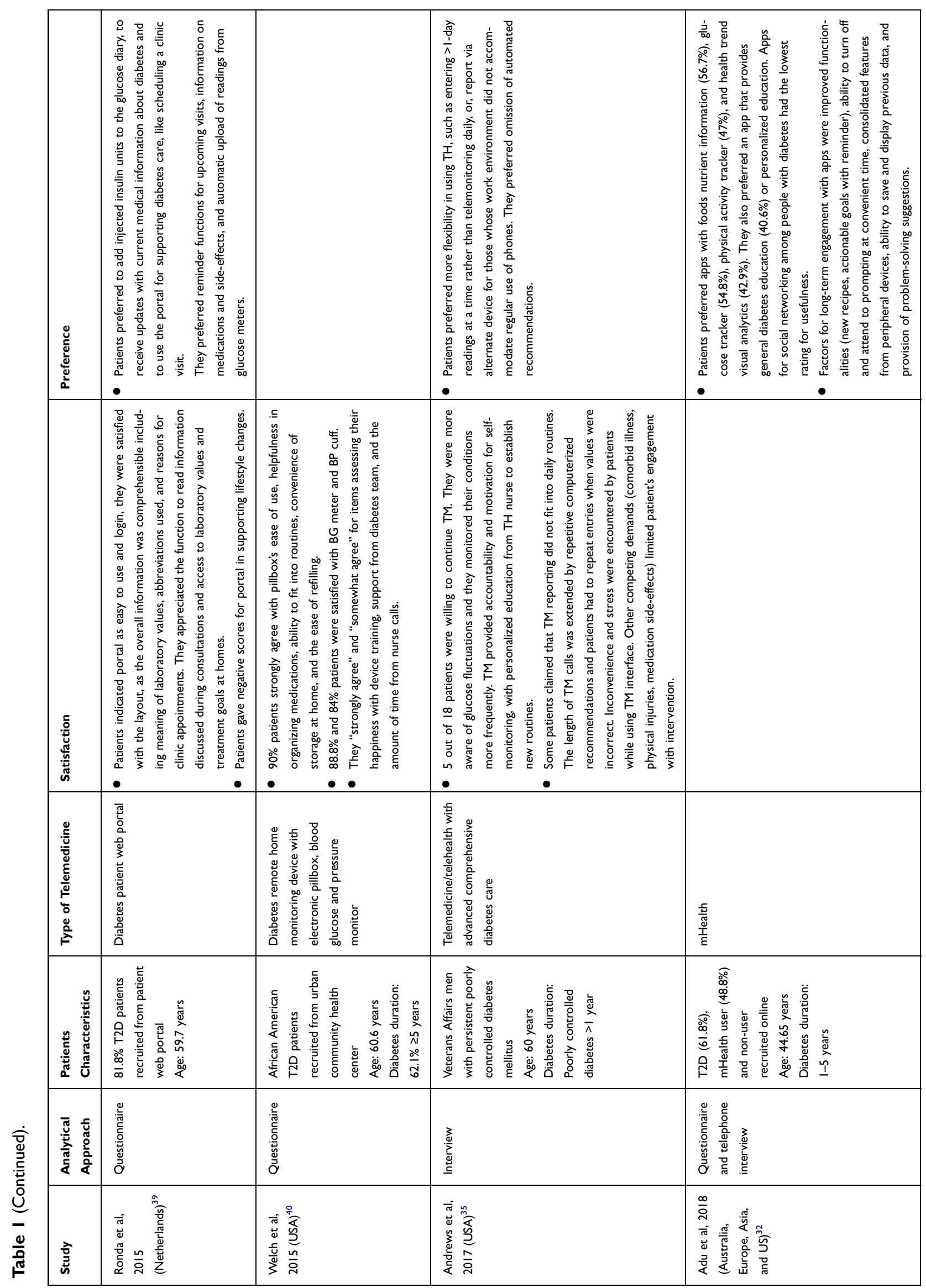




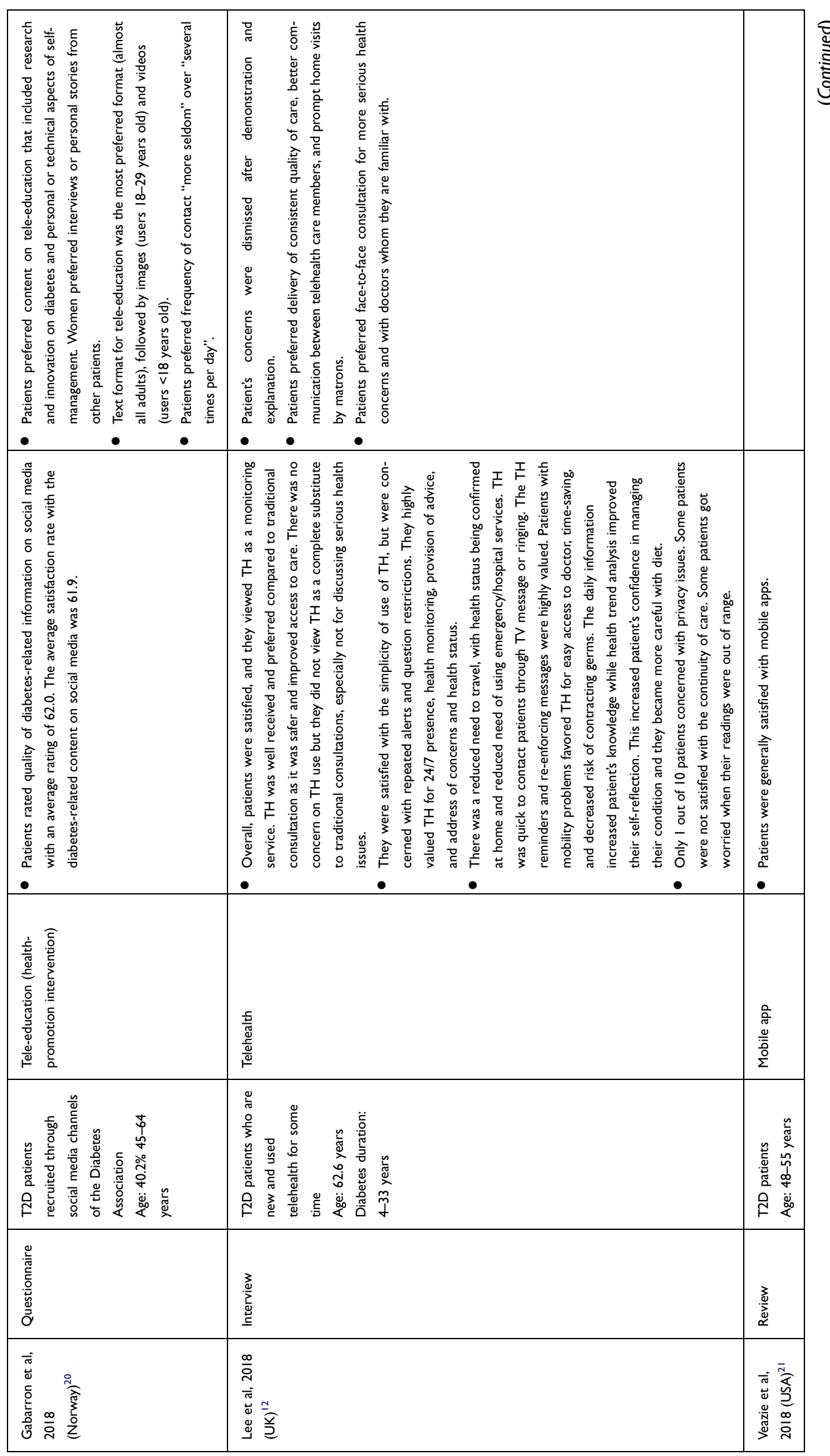




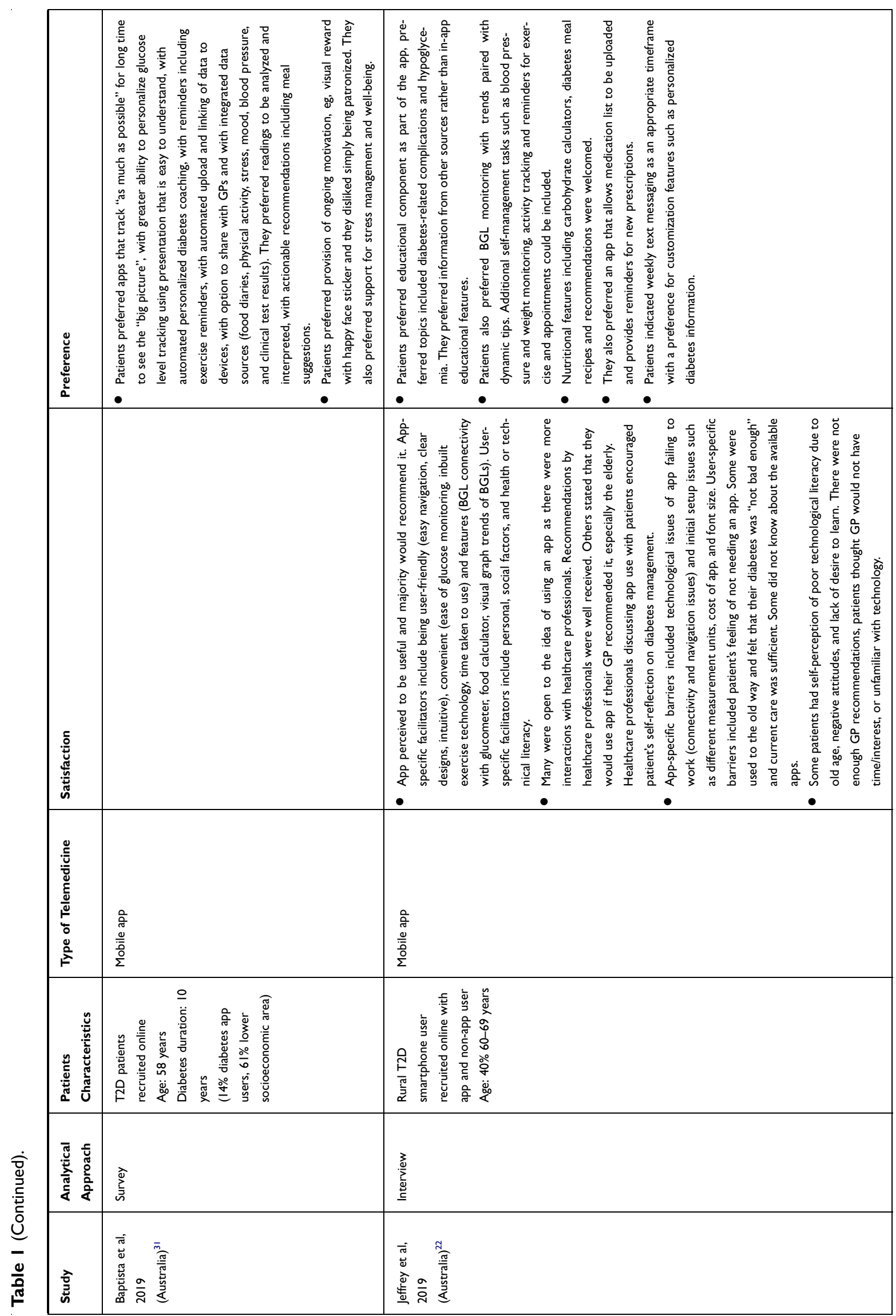




\begin{tabular}{|c|c|c|}
\hline 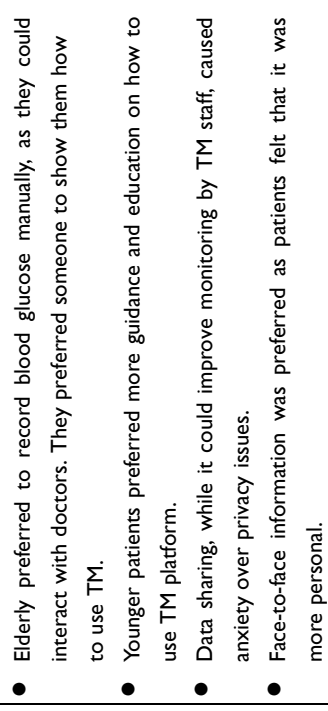 & 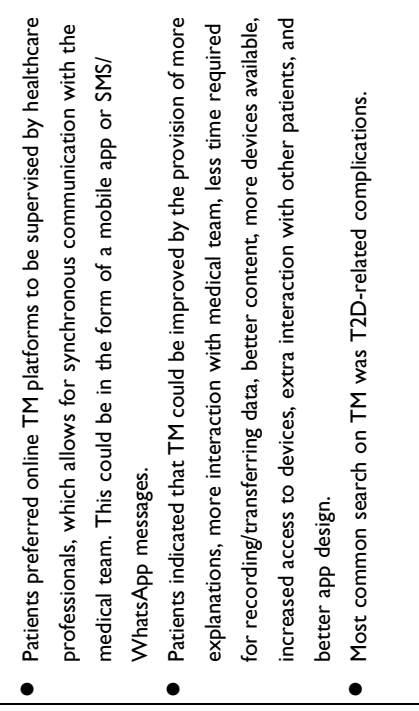 & 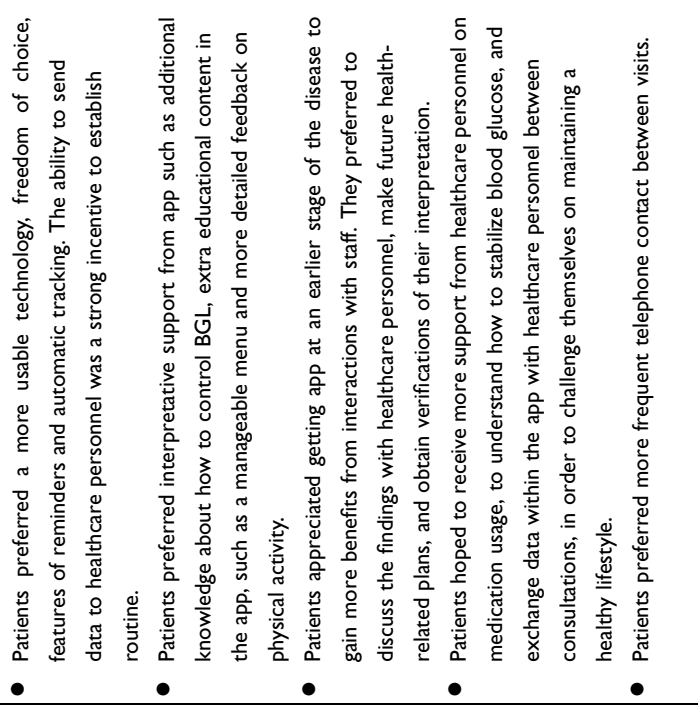 \\
\hline 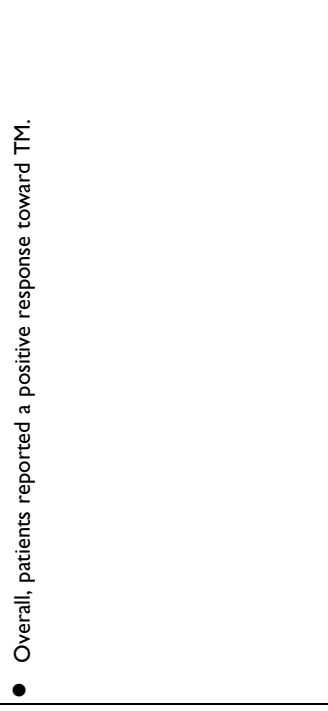 & 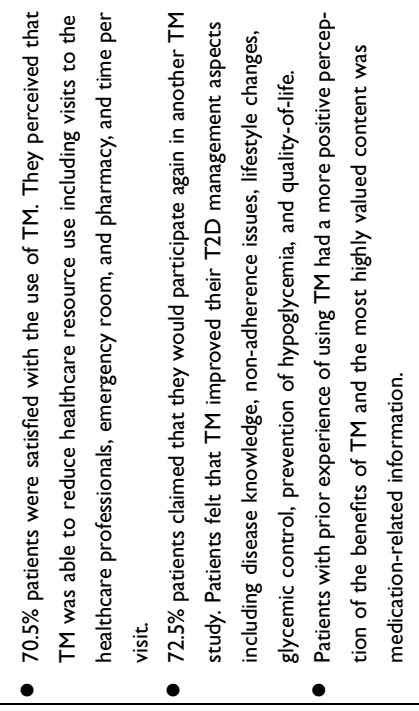 & 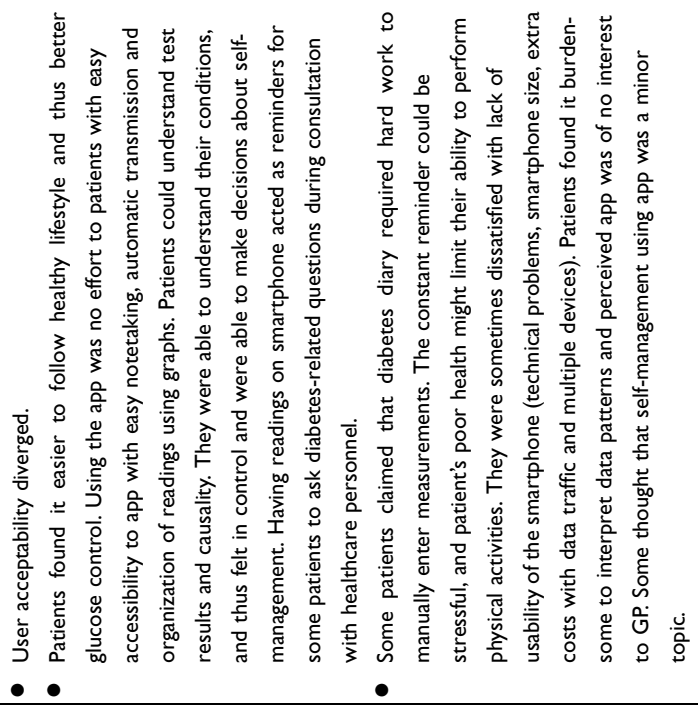 \\
\hline & & 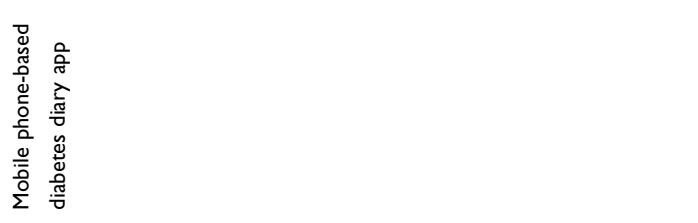 \\
\hline 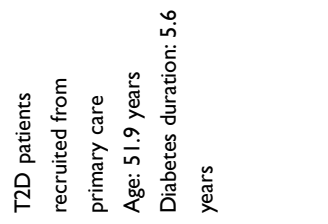 & 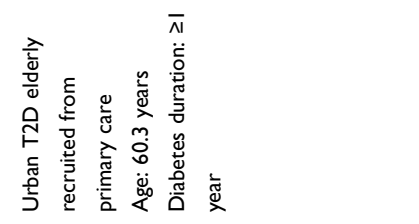 & 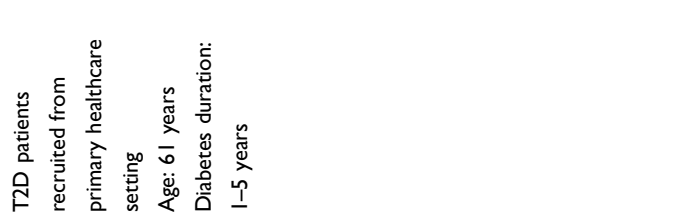 \\
\hline & 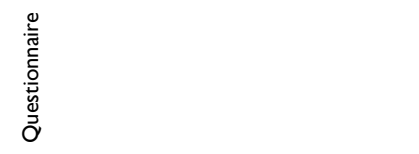 & 旁 \\
\hline 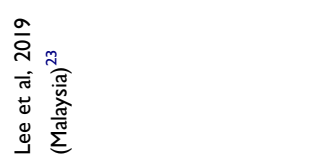 & 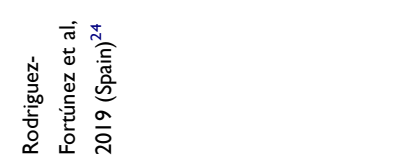 & 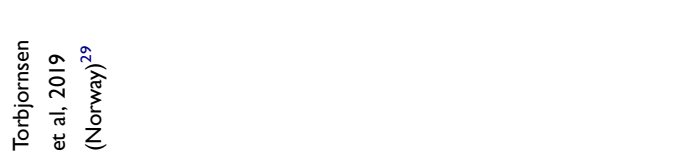 \\
\hline
\end{tabular}




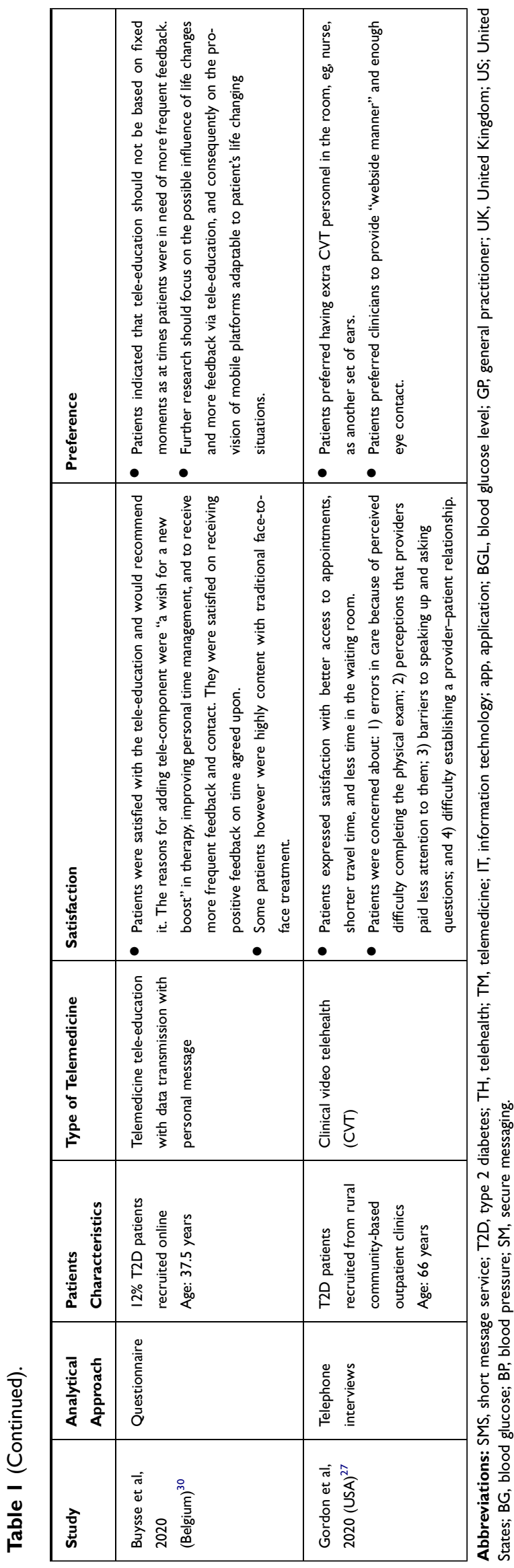

consultation, found it to be time-saving. ${ }^{12}$ Nevertheless, there were some concerns among patients with regard to the cost associated with telemedicine. Patients expressed dissatisfaction with the extra costs associated with use of data traffic and managing multiple devices and applications. $^{22,29}$

\section{Patient Preferences}

While patients have expressed satisfaction over the use of telemedicine, there are several factors which we found integral to ensure the continued use and success for any telemedicine related device. These features are summarized below.

\section{Education and Empowerment}

Patients often prefer face-to-face consultation, as they felt that information conveyed to them is clearer due to the ability to interact with the healthcare provider. ${ }^{23,30}$ Cognizant of this, trials on telemedicine to date have often adopted a model to ensure that there is a two-way communication between patients and the healthcare team. ${ }^{9,10}$ Studies have shown that among all the telemedicine strategies, teleconsultation with clinician involvement is the most effective strategy in reducing glycated hemoglobin. ${ }^{9}$ This is especially true for older adults, who stated that they preferred experts to show them how to use the technology. ${ }^{23,26}$ In the study by Yip et $\mathrm{al}^{17}$ in 2002, the lack of perceived need of assistance, together with the ability to meet healthcare needs, were the most important predictors of satisfaction for telemedicine. As such, it is important to conduct proper education and training for the patients. This can be delivered either through face-toface trainings or pre-recorded videos to eliminate the barriers toward adoption of telemedicine or technology that can be sometimes perceived as too technical.

For people with type 2 diabetes, diabetes education is the major driving factor in treatment satisfaction. ${ }^{34}$ As such, it is not surprising to note that patient's preference for telemedicine increased, especially for devices or platforms that incorporated diabetes education. ${ }^{20,22,24,29}$ Most patients were satisfied with the consistent and personalized education from healthcare providers in helping them to establish new routines. ${ }^{12,17,35}$ In a study by Lopez et al, ${ }^{36}$ they found that information given by healthcare professionals played a pivotal role in patient's inclination toward self-management of blood glucose. With the help of telemedicine, self-care behavioral changes can be integrated into a patient's routine. ${ }^{37}$ This, when coupled with the positive reinforcement features can further increase patient 
Table 2 Affinity Matrix

\begin{tabular}{|c|c|c|}
\hline Major Themes & Study Reference Number & Frequency \\
\hline \multicolumn{3}{|l|}{ Patient satisfaction } \\
\hline Round the clock access and monitoring & {$[12,22,23,25,29-32]$} & 8 \\
\hline Access to different healthcare providers & {$[12,22,24,25,27,29]$} & 6 \\
\hline Time-saving & {$[12,17,24,25,27]$} & 5 \\
\hline \multicolumn{3}{|l|}{ Patient preferences } \\
\hline Education and empowerment & {$[12,17,20,22-24,26,29-31,35,39]$} & 12 \\
\hline Support team & {$[16, \mid 8,20,24,27,29,31,32,40]$} & 9 \\
\hline Flexibility & {$[18,20,22,24,29-31,35]$} & 8 \\
\hline mHealth the choice & {$[22-24,29-32]$} & 7 \\
\hline Physician endorsement & {$[22,25,29]$} & 3 \\
\hline
\end{tabular}

satisfaction. $^{29,31,38}$ Some topics on self-management of blood glucose were well embraced, including diabetes complications, medication side-effects, as well as the latest findings in diabetes treatment and innovative therapies. $^{20,22,24,39}$

\section{Support Team}

We found that the rate of engagement of a telemedicine platform relies heavily on the support team who were managing the system. ${ }^{16,29,40}$ Younger patients expressed a preference for the team to comprise of knowledgeable staff who were also friendly and caring. ${ }^{18}$ They also expressed that staffneed to be more empathetic and acknowledged that while it is impossible to achieve perfect diabetes self-care, the team should provide positive reassurance and support to them during this journey. ${ }^{27,31}$ Some patients also expressed a preference for peer-support groups, as they felt that learning and sharing from other individuals' success stories in diabetes management motivated them. ${ }^{20,24}$ This preference, however, was not consistently expressed by all patients, suggesting the need for an individualized approach. ${ }^{24,32}$

\section{Flexibility}

When using telemedicine, patients preferred a system that was highly flexible, where they were able to customize the functions, messages, and alerts as needed. ${ }^{18,30,35}$ Patients described the importance of integrating multiple devices such as blood glucose monitors, smartphones, and electronic health records into a single platform, with the ability of automated uploading and synchronization of day-to-day data with the platform. ${ }^{24,29,31}$ Others suggested to include features such as allowing medication lists to be uploaded and medication collection reminders. ${ }^{22}$ Studies noted that it was important for the developers to note the different preferences and needs of different age groups. For example, text was the preferred mode of delivery for the older population while images or videos were preferred by the younger population. This emphasized the need for inclusion of customization and personalization features. ${ }^{20}$ These functions also need to be sufficiently discreet and not to intrude into the patient's life excessively. Examples of personalization of telemedicine include health advice given outside of patient's shift work hours or based on patient's existing exercise regimen. ${ }^{18,35}$ Indeed, patients found repeated alerts and "one answer per question" restrictions on the telemedicine platform frustrating. ${ }^{12,35}$ Constant alerts also resulted in "information fatigue" among patients, which caused some patients to report unnecessary stress. $^{29}$

\section{mHealth the Choice}

Most people expressed preference for a mobile application as opposed to online platforms when using any telemedicine modality. ${ }^{24,30}$ This is not surprising given that the rate of mobile phone ownership has increased by leaps and bounds over the past two decades. ${ }^{24,41,42}$ Patients indicated a preference for a "super application" that combined documentation, reminder, and advisory functions. ${ }^{32}$ Documentation features should include blood glucose logging, nutrition tracking, physical activity tracking, data sharing, and visualization. ${ }^{22,29,31,32}$ Reminder features should be tailored based on the data entered including self-care management tips, medication reminders, and exercise reminders. ${ }^{22}$ Patients appreciated reminders and reinforcing messages received through telemedicine platforms. $^{29}$ Advisory features of an application may include customized diabetes information for patients. In particular, patients referred to the need of an intuitive 


\section{What makes or breaks telemedicine for people with type 2 diabetes}
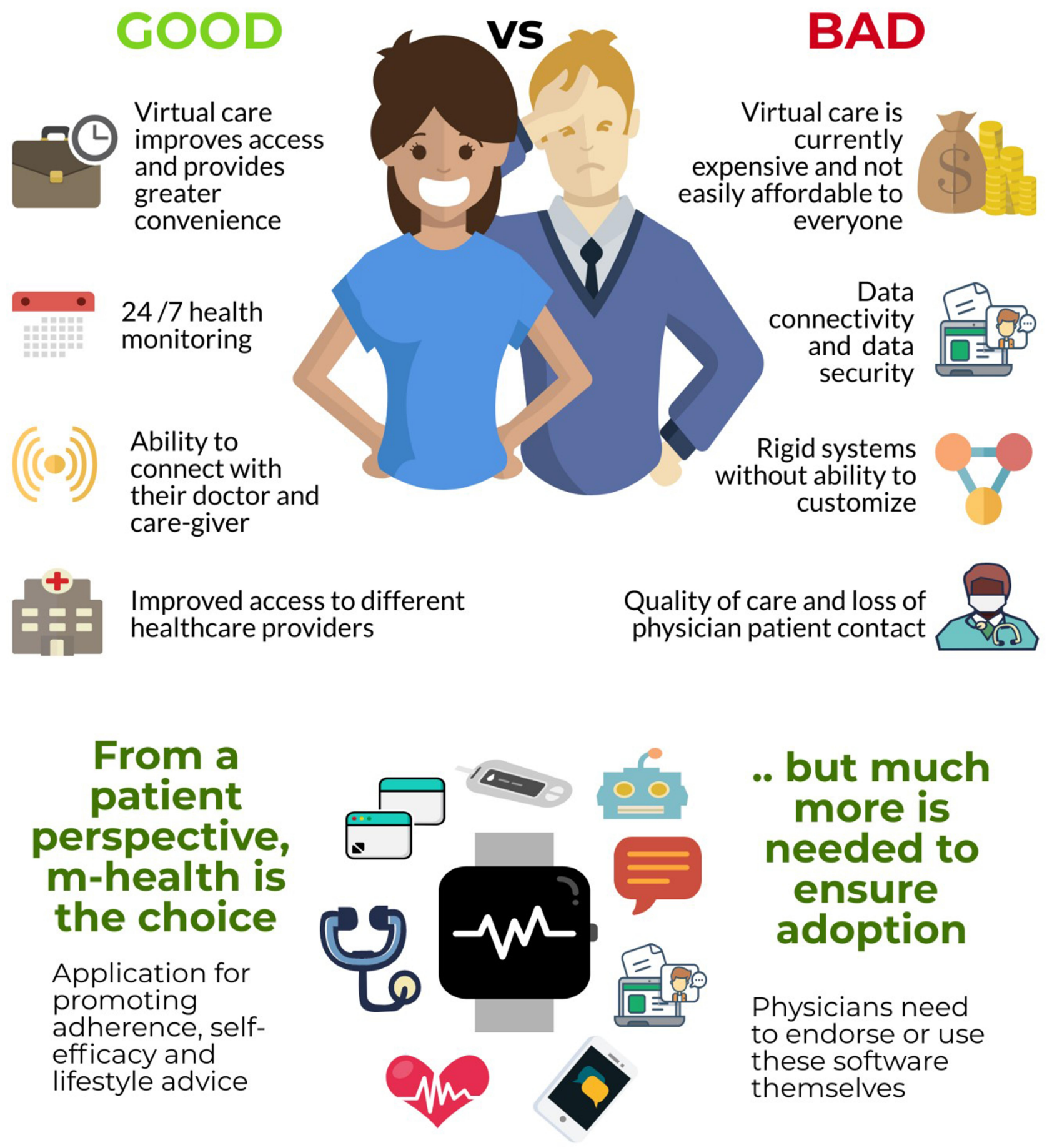

.. but much more is needed to ensure adoption

Physicians need to endorse or use these software themselves 
technology, with an automated personalized diabetes management coaching application depending on their glucose levels. $^{22,31}$ Patients also indicated a preference for mobile application that allows swifter access to healthcare practitioners. ${ }^{23}$ With the advancement of predictive technologies and artificial intelligence in telemedicine, this may be able to satisfy patient's need in the near future.

\section{Physician Endorsement}

Patients stated the preference to use an application if their general practitioner recommended it. ${ }^{22}$ When asked, some patients felt that their primary care physicians would not be interested, would not have time for, or were unfamiliar with telemedicine. ${ }^{2,25,29}$ Concerns were raised among chronic disease patients on the possibility of telemedicine causing depersonalized interactions with their physicians. ${ }^{43}$ Studies have shown that general practitioners frequently used applications for quick references but only a little more than half recommended applications for patients or discussed them. ${ }^{29,44,45}$ Patients appreciated obtaining a suitable mobile application at the earlier stage of disease to maximize the benefit gained. ${ }^{29}$

\section{Knowledge Gaps and Future Research}

We found that there was scant evidence examining the preference and satisfaction of patients with type 2 diabetes. Most of the current studies focused on patients with type 1 diabetes or examined quantitative outcomes such as glycated hemoglobin and adherence. ${ }^{9}$ Another gap identified in this review was the understanding of how patient's socio-economic factors, especially the marginalised population and ethnic minorities influenced patient's preference for telemedicine. Studies have shown that sociodemographic and socioeconomic factors can influence patient's usage of telemedicine. ${ }^{46,47}$ All the findings identified in this review were based on feedback from the general population, without a detailed understanding into the preferred modalities based on specific sociodemographic characteristics, eg, age, ethnicity, and income level. As the telemedicine usage is growing remarkably in the management of type 2 diabetes, future work should further expand to explore the views and preferences of patients from different sociodemographic groups. Researchers should also work on a standardized patient satisfaction rubric for telemedicine, and examine how these indicators may change over time with technological advancements.

The concern over additional costs incurred with telemedicine use has been identified in some studies as one of the factors affecting patient's adoption of telemedicine in diabetes. $^{22,48}$ However, this should be weighed against the benefits of cost savings from utilizing telemedicine in terms of traveling, waiting time, absence from work, and reduced hospitalization cost. ${ }^{49}$ Telemedicine providers should provide an insight into potential cost savings in adopting telemedicine compared to traditional approaches, on top of conventional efficacy measurement (eg, HbA1c). Our study also did not find any study elucidating patients' willingness to pay for a telemedicine platform. ${ }^{33}$ Future studies could focus on the economic aspect of adopting telemedicine as it has become more affordable and accessible.

In addition, the constantly evolving landscape in technology might act as a spur to changes in patient preference in the next few decades. The rapid development of telemedicine technologies seems to outpace research. Published studies suggest that the safety and/or effectiveness of any particular interventions and approval from accrediting bodies are among the chief concerns among physicians in advocating health technologies. ${ }^{50}$ Consequently, continuous research in this area is essential to ensure the appropriate application of telemedicine.

\section{Policy Implications}

All in all, patients appreciate the human aspect of telemedicine, especially when they feel that they are being understood and that their needs are being met. To encourage the use of telemedicine on a wider scale, the clinical outcomes, cost, availability, and user satisfaction need to be taken into account. ${ }^{51}$

Here we list down some recommendations for improvement in patient satisfaction.

1. Endorsement of healthcare providers, including physicians and diabetes educators, plays an important role in the adoption of telemedicine. It might be beneficial to look into ways to increase interest of healthcare practitioners toward telemedicine. This could be achieved through a collaboration between service providers, developers, and healthcare practitioners in this area. It is also important to arrange for appropriate reimbursement for physicians to deliver care in the form of telemedicine. Healthcare regulators or accreditation bodies should attempt to provide a list of safe and effective telemedicine interventions such as the NHS Apps Library. The formation of digital formularies by 
professional regulatory bodies ensures that patients and physicians are not lost in the wild array of telemedicine devices and raises their trust for telemedicine.

2. Ample technical guidance should be given to promote the seamless transition from conventional consultation into telemedicine. Knowing that patients are receptive to the idea of telemedicine, healthcare providers should empower patients with the technical knowledge to use telemedicine. Diligent and enthusiastic participation of clinicians and the telemedicine care team is more likely to lead to better patient engagement. Healthcare providers should emphasize the high return on the time and cost by undertaking telemedicine.

3. Consistent and continuous diabetes education should continue to be delivered using telemedicine. Physicians should help patients recognize the severity of their disease and the potential advantages of telemedicine in managing their blood glucose in a realistic manner. Healthcare providers have to be equipped with not just technical competence, but also proper information delivery through appropriate body language and eye contact. ${ }^{13,27}$

4. mHealth should be integrated into different telemedicine modalities as the majority of healthcare providers and patients have access to ICT in the form of mobile phones. ${ }^{24,44,45}$ The delivery of healthcare should not be limited to a particular form of technology but should be diversified through alternate forms of technology that best suit the patient. This is especially true in rural areas where innovation should take place for telemedicine modality selection. Instead of viewing the digital divide as an obstacle to telemedicine, technology should be fully utilized for healthcare to reach the underserved group at a greater scale.

5. Lastly, granted there will not be a one size fits all solution, future telemedicine design should move toward a personalized approach based on the patient's condition. With the advancement of predictive technologies, telemedicine products are increasingly intuitive and humanized. Artificial intelligence products including diabetes chatbots have been developed for better patient education. ${ }^{52}$ It is important to gather adequate feedback, especially in the area of feeling and perspective from patients so that adequate human touch can be instilled into the technologies while attaining realistic goals. ${ }^{52}$ Telemedicine providers should also work toward integrating patient data into their services and harness the power of cloud computing for population data storage and flexible user access. Certainly, proper due diligence should be carried out to safeguard patient's personal details so that their trust will not be jeopardized.

\section{Strengths and Limitations}

To the best of our knowledge this is the first narrative review that examined satisfaction and preference of patients with type 2 diabetes toward telemedicine. This study adds to the growing literature on the effective use of telemedicine in managing type 2 diabetes as telemedicine use has been increasing exponentially during the COVID-19 pandemic. $^{53,54}$ There are some limitations to our review. Firstly, this review was intended as a narrative review rather than a systematic review, hence possibilities of selection bias should be considered. While studies of some technologies were more frequently reported, eg, mHealth, others were less studied, eg, game-based support. However, we have searched across three large databases and included all types of technologies that were reported. Secondly, even though the telemedicine strategies were grouped into different modalities, there were still significant differences in the implementation details within each modality, eg, the level of engagement of healthcare practitioners. Due to the limited number of studies, we did not attempt to stratify patient satisfaction and preference based on different age groups and socioeconomic factors. Furthermore, the measurement methods for patient satisfaction were largely heterogenous, compounded by different stakeholders' interest, thereby limiting comparison across studies.

\section{Concluding Remarks}

Telemedicine has come a long way from being a concept to a science-based reality. In realising the full potential of this reality, the patient remains at the center of telemedicine design in managing type 2 diabetes. Healthcare providers should cease underestimating the patient's ability to engage with technology and move toward improving the existing technologies to be more streamlined and adaptive according to patient preference. With proper evaluation and recommendation, telemedicine will be one of the of best tools to provide quality healthcare to all patients with type 2 diabetes. 


\section{Author Contributions}

All authors contributed to data analysis, drafting or revising the article, have agreed on the journal to which the article will be submitted, gave final approval of the version to be published, and agree to be accountable for all aspects of the work.

\section{Disclosure}

The authors report no conflicts of interest in this work.

\section{References}

1. International Diabetes Federation. IDF Diabetes Atlas. 9th Edition. 2019.

2. Roth GA, Abate D, Abate $\mathrm{KH}$, et al. Global, regional, and national age-sex-specific mortality for 282 causes of death in 195 countries and territories, 1980-2017: a systematic analysis for the global burden of disease study 2017. Lancet. 2018;392(10159):1736-1788.

3. American Diabetes Association. Implications of the United Kingdom prospective diabetes study. Diabetes Care. 2002;25(suppl1):s28-s32. doi:10.2337/diacare.25.2007.S28

4. King P, Peacock I, Donnelly R. The UK prospective diabetes study (UKPDS): clinical and therapeutic implications for type 2 diabetes. Br J Clin Pharmacol. 1999;48(5):643-648. doi:10.1046/j.13652125.1999.00092.x

5. Rodríguez-Gutiérrez R, Montori VM. Glycemic control for patients with type 2 diabetes mellitus: our evolving faith in the face of evidence. Circ Cardiovasc Qual Outcomes. 2016;9(5):504-512. doi:10.1161/CIRCOUTCOMES.116.002901

6. Harris M. Challenges in diabetes management. Aust Fam Physician. 2008;37(9):716-720

7. Association AT. Telehealth basics. Available from: https://www.amer icantelemed.org/resource/why-telemedicine/. Accessed August 1, 2020

8. Lee SWH, Ooi L, Lai YK. Telemedicine for the management of glycemic control and clinical outcomes of type 1 diabetes mellitus: a systematic review and meta-analysis of randomized controlled studies. Front Pharmacol. 2017;8:330. doi:10.3389/fphar.2017.00330

9. Lee SWH, Chan CKY, Chua SS, Chaiyakunapruk N. Comparative effectiveness of telemedicine strategies on type 2 diabetes management: a systematic review and network meta-analysis. Sci Rep. 2017;7(1):12680. doi:10.1038/s41598-017-12987-z

10. Chakranon P, Lai YK, Tang YW, Choudhary P, Khunti K, Lee SWH. Distal technology interventions in people with diabetes: an umbrella review of multiple health outcomes. Diabet Med. 2020;37(12):19661976. doi:10.1111/dme. 14156

11. Chongmelaxme B, Lee $\mathrm{S}$, Dhippayom $\mathrm{T}$, Saokaew $\mathrm{S}$, Chaiyakunapruk N, Dilokthornsakul P. The effects of telemedicine on asthma control and patients' quality of life in adults: a systematic review and meta-analysis. J Allergy Clin Immunol Pract. 2019;7 (1):199-216.e111. doi:10.1016/j.jaip.2018.07.015

12. Lee P, Greenfield G, Pappas Y. Patients' Perception of Using Telehealth for Type 2 Diabetes Management: A Phenomenological Study. 2018.

13. Barbosa CD, Balp -M-M, Kulich K, Germain N, Rofail D. A literature review to explore the link between treatment satisfaction and adherence, compliance, and persistence. Patient Prefer Adherence. 2012;6:39-48. doi:10.2147/PPA.S24752

14. Duke DC, Barry S, Wagner DV, Speight J, Choudhary P, Harris MA. Distal technologies and type 1 diabetes management. Lancet Diabetes Endocrinol. 2018;6(2):143-156. doi:10.1016/S2213-8587 (17)30260-7
15. Fleming GA, Petrie JR, Bergenstal RM, Holl RW, Peters AL, Heinemann L. Diabetes digital app technology: benefits, challenges, and recommendations. a consensus report by the European association for the study of diabetes (EASD) and the American diabetes association (ADA) diabetes technology working group. Diabetologia. 2020;63(2):229-241. doi:10.1007/s00125-019-05034-1

16. Siminerio L, Ruppert K, Huber K, Toledo FG. Telemedicine for reach, education, access, and treatment (TREAT): linking telemedicine with diabetes self-management education to improve care in rural communities. Diabetes Educ. 2014;40(6):797-805. doi:10.1177/0145721714551993

17. Yip MP, Mackenzie A, Chan J. Patient satisfaction with telediabetes education in Hong Kong. J Telemed Telecare. 2002;8(1):48-51. doi:10.1258/1357633021937460

18. Long AF, Gambling T, Young RJ, Taylor J, Mason JM. Acceptability and satisfaction with a telecarer approach to the management of type 2 diabetes. Diabetes Care. 2005;28(2):283-289. doi:10.2337/ diacare.28.2.283

19. Georgsson M, Staggers N. Quantifying usability: an evaluation of a diabetes mHealth system on effectiveness, efficiency, and satisfaction metrics with associated user characteristics. J Am Med Inform Assoc. 2016;23(1):5-11. doi:10.1093/jamia/ocv099

20. Gabarron E, Dorronzoro E, Bradway M, Rivera-Romero O, Wynn R, Årsand E. Preferences and interests of diabetes social media users regarding a health-promotion intervention. Patient Prefer Adherence. 2018;12:2499-2506. doi:10.2147/PPA.S184369

21. Veazie SWK, Gilbert J, Paynter R, et al. Mobile Applications for SelfManagement of Diabetes. Technical Brief No. 31. (Prepared by the Scientific Resource Center Under Contract Nos. 290-2012-0004-C and 290-2017-00003-C). AHRQ Publication No 18-EHC010-EF Rockville, MD. Agency for Healthcare Research and Quality; 2018.

22. Jeffrey B, Bagala M, Creighton A, et al. Mobile phone applications and their use in the self-management of type 2 diabetes mellitus: a qualitative study among app users and non-app users. Diabetol Metab Syndr. 2019;11(1):84. doi:10.1186/s13098-019-0480-4

23. Lee JY, Chan CKY, Chua SS, et al. Using telemedicine to support care for people with type 2 diabetes mellitus: a qualitative analysis of patients' perspectives. BMJ Open. 2019;9(10):e026575-e026575. doi:10.1136/bmjopen-2018-026575

24. Rodríguez-Fortúnez P, Franch-Nadal J, Fornos-Pérez JA, MartínezMartínez F, de Paz HD, Orera-Peña ML. Cross-sectional study about the use of telemedicine for type 2 diabetes mellitus management in Spain: patient's perspective. The EnREDa2 study. BMJ Open. 2019;9 (6):e028467. doi:10.1136/bmjopen-2018-028467

25. Wade-Vuturo AE, Mayberry LS, Osborn CY. Secure messaging and diabetes management: experiences and perspectives of patient portal users. J Am Med Inform Assoc. 2012;20(3):519-525. doi:10.1136/ amiajnl-2012-001253

26. Rho MJ, Kim SR, Kim HS, et al. Exploring the relationship among user satisfaction, compliance, and clinical outcomes of telemedicine services for glucose control. Telemed J E Health. 2014;20(8):712720. doi:10.1089/tmj.2013.0309

27. Gordon HS, Solanki P, Bokhour BG, Gopal RK. I'm not feeling like i'm part of the conversation patients' perspectives on communicating in clinical video telehealth visits. J Gen Intern Med. 2020;35 (6):1751-1758. doi:10.1007/s11606-020-05673-w

28. Center PR. Tech Adoption Climbs Among Older Adults. 2017.

29. Torbjørnsen A, Ribu L, Rønnevig M, Grøttland A, Helseth S. Users' acceptability of a mobile application for persons with type 2 diabetes: a qualitative study. BMC Health Serv Res. 2019;19(1):641. doi:10.1186/s12913-019-4486-2

30. Buysse H, Coremans P, Pouwer F, Ruige J. Sustainable improvement of $\mathrm{HbA} 1 \mathrm{c}$ and satisfaction with diabetes care after adding telemedicine in patients on adaptable insulin regimens: results of the TeleDiabetes randomized controlled trial. Health Informatics J. 2019;26(1):628-641. doi:10.1177/1460458219844369 
31. Baptista S, Trawley S, Pouwer F, Oldenburg B, Wadley G, Speight J. What do adults with type 2 diabetes want from the "perfect" app? Results from the second diabetes MILES: Australia (MILES-2) study. Diabetes Technol Ther. 2019;21(7):393-399. doi:10.1089/ dia.2019.0086

32. Adu MD, Malabu UH, Malau-Aduli AEO, Malau-Aduli BS. Users' preferences and design recommendations to promote engagements with mobile apps for diabetes self-management: multi-national perspectives. PLoS One. 2018;13(12):e0208942. doi:10.1371/journal. pone.0208942

33. Lee JY, Lee SWH. Telemedicine cost-effectiveness for diabetes management: a systematic review. Diabetes Technol Ther. 2018;20 (7):492-500. doi:10.1089/dia.2018.0098

34. Boels AM, Vos RC, Hermans TGT, et al. What determines treatment satisfaction of patients with type 2 diabetes on insulin therapy? An observational study in eight European countries. BMJ Open. 2017;7 (7):e016180. doi:10.1136/bmjopen-2017-016180

35. Andrews SM, Sperber NR, Gierisch JM, et al. Patient perceptions of a comprehensive telemedicine intervention to address persistent poorly controlled diabetes. Patient Prefer Adherence. 2017;11:469478. doi:10.2147/PPA.S125673

36. Lopez JMS, Katic BJ, Fitz-Randolph M, Jackson RA, Chow W, Mullins CD. Understanding preferences for type 2 diabetes mellitus self-management support through a patient-centered approach: a 2phase mixed-methods study. BMC Endocr Disord. 2016;16(1):41. doi: 10.1186/s12902-016-0122-X

37. Kebede MM, Pischke CR, Popular D. Apps and the impact of diabetes app use on self-care behaviour: a survey among the digital community of persons with diabetes on social media. Front Endocrinol (Lausanne). 2019;10:135. doi:10.3389/fendo.2019.00135

38. McMillan KA, Kirk A, Hewitt A, MacRury S. A systematic and integrated review of mobile-based technology to promote active lifestyles in people with type 2 diabetes. J Diabetes Sci Technol. 2017;11 (2):299-307. doi:10.1177/1932296816656018

39. Ronda MCM, Dijkhorst-Oei L-T, Rutten GEHM. Patients' experiences with and attitudes towards a diabetes patient web portal. PLoS One. 2015;10(6): $0129403-\mathrm{e} 0129403 . \quad$ doi:10.1371/journal. pone. 0129403

40. Welch G, Balder A, Zagarins S. Telehealth program for type 2 diabetes: usability, satisfaction, and clinical usefulness in an urban community health center. Telemed J E Health. 2015;21(5):395-403. doi:10.1089/tmj.2014.0069

41. El-Gayar O, Timsina P, Nawar N, Eid W. Mobile applications for diabetes self-management: status and potential. J Diabetes Sci Technol. 2013;7(1):247-262. doi:10.1177/193229681300700130
42. Center PR. Mobile Technology and Home Broadband 2019. 2019.

43. Martinez PR. A Qualitative Study on Patient Perceptions Towards mHealth Technology Among High Risk, Chronic Disease Patients. 2015.

44. Byambasuren O, Beller E, Glasziou P. Current knowledge and adoption of mobile health apps among Australian general practitioners: survey study. JMIR Mhealth Uhealth. 2019;7(6):e13199-e13199. doi: $10.2196 / 13199$

45. Liu Y, Ren W, Qiu Y, Liu J, Yin P, Ren J. The use of mobile phone and medical apps among general practitioners in Hangzhou City, Eastern China. JMIR Mhealth Uhealth. 2016;4(2):e64-e64. doi: $10.2196 /$ mhealth. 4508

46. Sun R, Burke LE, Saul MI, Korytkowski MT, Li D, Sereika SM. Use of a patient portal for engaging patients with type 2 diabetes: patterns and prediction. Diabetes Technol Ther. 2019;21(10):546-556. doi:10.1089/dia.2019.0074

47. Reed ME, Huang J, Graetz I, et al. Patient characteristics associated with choosing a telemedicine visit vs office visit with the same primary care clinicians. JAMA Netw Open. 2020;3(6):e205873e205873. doi:10.1001/jamanetworkopen.2020.5873

48. Park H, Chon Y, Lee J, Ie J C, Yoon KH. Service design attributes affecting diabetic patient preferences of telemedicine in South Korea. Telemed J E Health. 2011;17(6):442-451. doi:10.1089/tmj.2010.0201

49. Röhling M, Redaélli M, Simic D, et al. TeDia - a telemedicine-based treatment model for inpatient and interprofessional diabetes care. Diabetes Metab Syndr Obes. 2019;12:2479-2487. doi:10.2147/ DMSO.S229933

50. Leigh S, Ashall-Payne L. The role of health-care providers in mHealth adoption. Lancet Glob Health. 2019;1(2):e58-e59. doi:10.1016/S2589-7500(19)30025-1

51. Nguyen M, Waller M, Pandya A, Portnoy JA. Review of patient and provider satisfaction with telemedicine. Curr Allergy Asthma Rep. 2020;20(11):72. doi:10.1007/s11882-020-00969-7

52. Unnikrishnan AG. Artificial intelligence in health care: focus on diabetes management. Indian J Endocrinol Metab. 2019;23(5):503506. doi:10.4103/ijem.IJEM 54919

53. Doraiswamy S, Abraham A, Mamtani R, Cheema S. Use of telehealth during the COVID-19 pandemic: scoping review. J Med Internet Res. 2020;22(12):e24087. doi:10.2196/24087

54. Koonin L, Hoots B, Tsang C, et al. Trends in the use of telehealth during the emergence of the COVID-19 pandemic - United States, january-march 2020. MMWR Morb Mortal Wkly Rep. 2020;69 (43):1595-1599. doi:10.15585/mmwr.mm6943a3
Patient Preference and Adherence

\section{Publish your work in this journal}

Patient Preference and Adherence is an international, peer-reviewed, open access journal that focusing on the growing importance of patient preference and adherence throughout the therapeutic continuum. Patient satisfaction, acceptability, quality of life, compliance, persistence and their role in developing new therapeutic modalities and compounds to optimize clinical outcomes for existing disease states are major areas of interest for the journal. This journal has been accepted for indexing on PubMed Central. The manuscript management system is completely online and includes a very quick and fair peer-review system, which is all easy to use. Visit http:// www.dovepress.com/testimonials.php to read real quotes from published authors. 